\title{
Cutaneous coding of optical signals: The optohapt'
}

FRANK A, GELDARD

PRINCETON UNIVERSITY

Means of transmuting optical signals into cutaneous pattems are sufficiently rare to warrant a fresh attempt at devising one. An instrument called the "optohapt" is described. It converts printed or typed characters inco tactual signals having various spatial and temporal properties. These are impressed on nine widely scattered bodily loci. Data on discriminability of a wide range of pacterns are reported, and there is proposed a promising coding system for the instrument.

In his celebrated educational treatise, Emile, Jean Jaques Rousseau made the observation, perhaps original with him, that man's sense of touch was so constituted that it should be possible for him to learn to feel musical sounds and to construct a tactual language from them. A little over two centuries ago Rousseau penned this imaginative passage:

"As our sense of feeling, when properly exercised, becomes a supplement to sight, why may it not also substitute for hearing to a certain degree, since sounds excite in resonant bodies vibrations sensibie to touch? Lay a hand on the body of the cello, and you will beable, without the assistance of either eyes or ears, to distinguish, merely by the way in which the wood vibrates and trembles, whether the sound it gives is deep or shrill, whether it comes from the treble or the bass. If one were to train the senses to these differences, I do not doubt that, in time, one could become so sensitive as to be able to distinguish a whole air by means of the fingers. Now, if we concede this, it is clear that we might easily talk to deaf people by means of music; for tone and measures are no less susceptible of regular combination than voice and articulation, so they may be made use of in the same way as the elements of speech." (Rousseau, 1762, pp. 237-238).

More than a century and a half later, psychology meanwhile having come of age and having acquired the accoutrements of the laboratory, the suggestion reappeared in various forms, and became a plan of experimental action. Both musical and speech sounds were led to electromechanical transducers and these were applied, chiefly to the sensitive finger tips, in the hope that the complex vibratory patterns thus transmitted would, through training, recreate their messages. One might be able to learn to "hear through the skin."

This is not the place to chronicle this effort and the disappointment ultimately attending it. Tactual stimuli generated in such a manner we now know not to fall within the skin's discrimination capacities, so all such approaches fail. But the dimmed hopes of the 1920's and $1930^{\prime} \mathrm{s}$ are perhaps forgotten in the $1960^{\prime} \mathrm{s}$, for the approach to cutaneous communication by way of complex, articulated arrays of stimuli impressed in toto on the skin characterizes several recent and current programs of research in this area. Contacting elements in the form of matrices of solenoids, crystal bimorphs, air jets, and electrodes are being employed, sometimes quite ingeniously, to create patterned displays which, through successions of pulses or vibratory bursts, deliver their information to the skin.

The older effort made use of auditory signals; many current attempts appeal to their visual analogs. This is perhaps a step forward, for somesthesis is a spatial sense, like vision, as well as a temporal one, like audition. Indeed, in a somewhat limited low-frequency range, the skin rivals the ear in making frequency discriminations, though in no range does it seriously compete with the ear as a mediator of intensitive differences. Similarly, there may be some as yet undisclosed spatial property of the skin that permits it to be compared favorably with the retina, but the simplest considerations dictate that the skin will, in general, always remain a poor relation of the eye in resolving spatial patterns while, at the same time, excelling it in the temporal domain.

Over an extended period the author has contended that the approach to cutaneous communication systems must be that of first ascertaining what discriminations the skin can make with relative ease. Then, by suitable engineering of the effective stimuli, coupled with appropriate coding to transmute the information to be conveyed, there needs to be worked out an orderly set of signals that falls within the skin's "'language," so to speak.

Over the years many of the basic discriminations have been charted. We are currently in possession of many useful facts about the skin's reception of stimulus intensity, duration, frequency, and even some of the details of the manner in which the spatial variable, locus, operates (Geldard, 1960). But it is this local mode of sensitivity variation that most urgently needs study. Only relatively recently have there been applied to widespread bodily areas multiple arrays of transducers capable of differential operation, and this is so because only recently have there been made available for such study the appropriate transducers (Bice, 1961; Sherrick, 1965).

Highly spatially dispersed patterns created by miniature vibrators have been utilized in a systematic way in the Princeton Cutaneous Communication Laboratory (Geldard \& Sherrick, 1965). These patterns, varying in number of elements from one to nine and involving 
skin areas selected for their probable "uniqueness" of contribution, could be generated in more than a thousand combinations; paired for comparison with one another it was possible to present well over a halfmillion different arrangements. But such patterns, generated as they were by slowly-rumning punched tapes, were relatively static in nature. All vibrators involved in a given pattern were energized simultaneously for a 200-msec, exposure and were cut off simultaneousiy at the end of that period. The capacity of the skin for making relatively fine temporal distinctions was not being utilized to differentiate patterns.

It would be possible to overcome this defect, of course, by utilizing a fast-running tape accommodating many channels. It could then be arranged, for any desired signal burst, to terminate some elements early and prolong others. Indeed, for a completely systematic approach to spatiotemporal patterning on the skin a multi-channel, suitably punched fast tape provides what appears to be the ideal vehicle. This Laboratory has some plans for such programming.

Meanwhile, there are some other possibilities. One of the more intriguing presented itself in the form of the Battelle Optophone. This instrument, lineal descendant of an earlier invention (Fournier d'Albe, 1914) has had a not too rewarding career as a device to assist the blind to "read." The central component of the instrument is a normally hand-held scanning probe housing a linear array of nine cadmium selenide photoconductive cells deposited on an alumina substrate. An optical system, which also provides illumination to the printed page (to be "read") can differentiate with some precision black marks on white paper (or the reverse). Printed characters, from 6- to 14-point, can be accommodated by the optical system; a simple calibration screw adjusts the image of a letter to cover the nine-cell array exactly. In its original use, when a black portion of a letter fell on a photocell there was generated one of nine tones, heard monaurally, arranged approximately in musical thirds from 440 to $2795 \mathrm{~Hz}$, the pitch number being roughly proportional to the height of that part of the letter above the base line. As Freiberger has described it (Freiberger, 1965): "... what the machine does is to erform a transformation from the $x-y$ display of ink on a page that constitutes printing, to a pitch-duration display which conveys the indicia information to the brain via the auditory sense."

The Battelle form of the optophone is still under test as a practical reading aid for the blind, but even its most ardent enthusiast will admit of some genuine difficulties to be overcome. The chief one, of course, is the "unnaturalness" of translating spatial position of a portion of a letter stroke into tonal pitch. Whereas it has to be admitted that there is some inherent "compatibility" between visual "up-down" and "high-low" pitch (Pratt, 1930), the systematic translation of lines into musical intervals, especially when they necessarily dance about and interweave with each other in a most bewildering fashion, is something else again. The simple fact is that onky in an entirely oblique and derived way is hearing a spatial sense. We know about auditory space only through localization (time-intensity) judgments and volumic-densitive appearances. To ask that visual spatial distinctions be apprehended in terms of patterns of pitches is to do violence to first psychological principles.

It does not seem improbable, on the other hand, that the only truly spatial sense, other than vision, that the body possesses might ultimately be able to perform the desired transmutation. It might be possible to convert the differential optophone output into a series of haptically perceived spatial distinctions. Indeed, there is already one effort to do so, the "visotactor" designed by Mauch Laboratories (Smith \& Mauch, 1964). This instrument derives from the optophone in a succession of engineering steps. At least two models are under development, one having an eight-vibrator tactile output, the other being a "multicolumn" version with 24 vibrators. The contactors, each controlled by a photocell, are embedded in the handle of the instrument and vibrate at $150 \mathrm{~Hz}$ against the fingers. There is no intermediate coding. The hope is that the somewhat elaborate spatiotemporal patterns created in the fingers as the optical system "looks at" print will, through

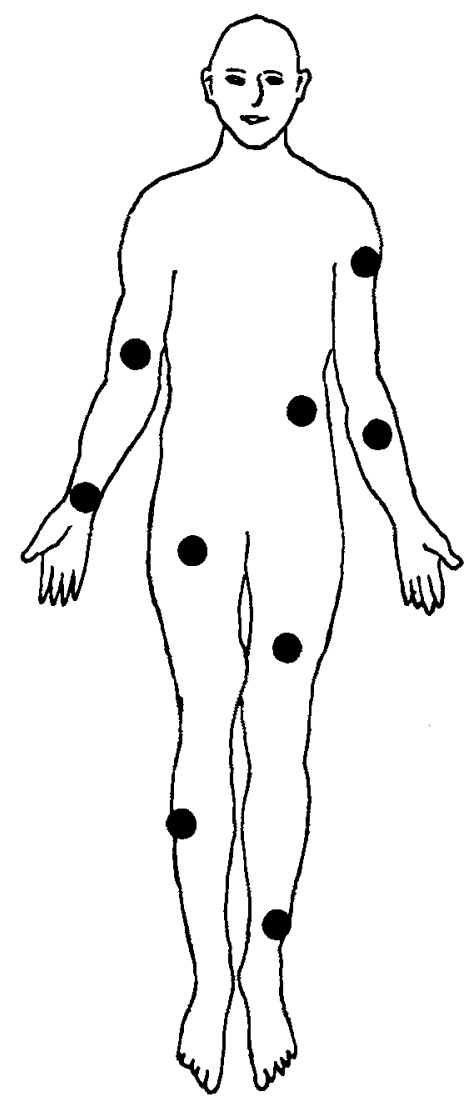

Fig. 1. Location of the nine vibrators. 


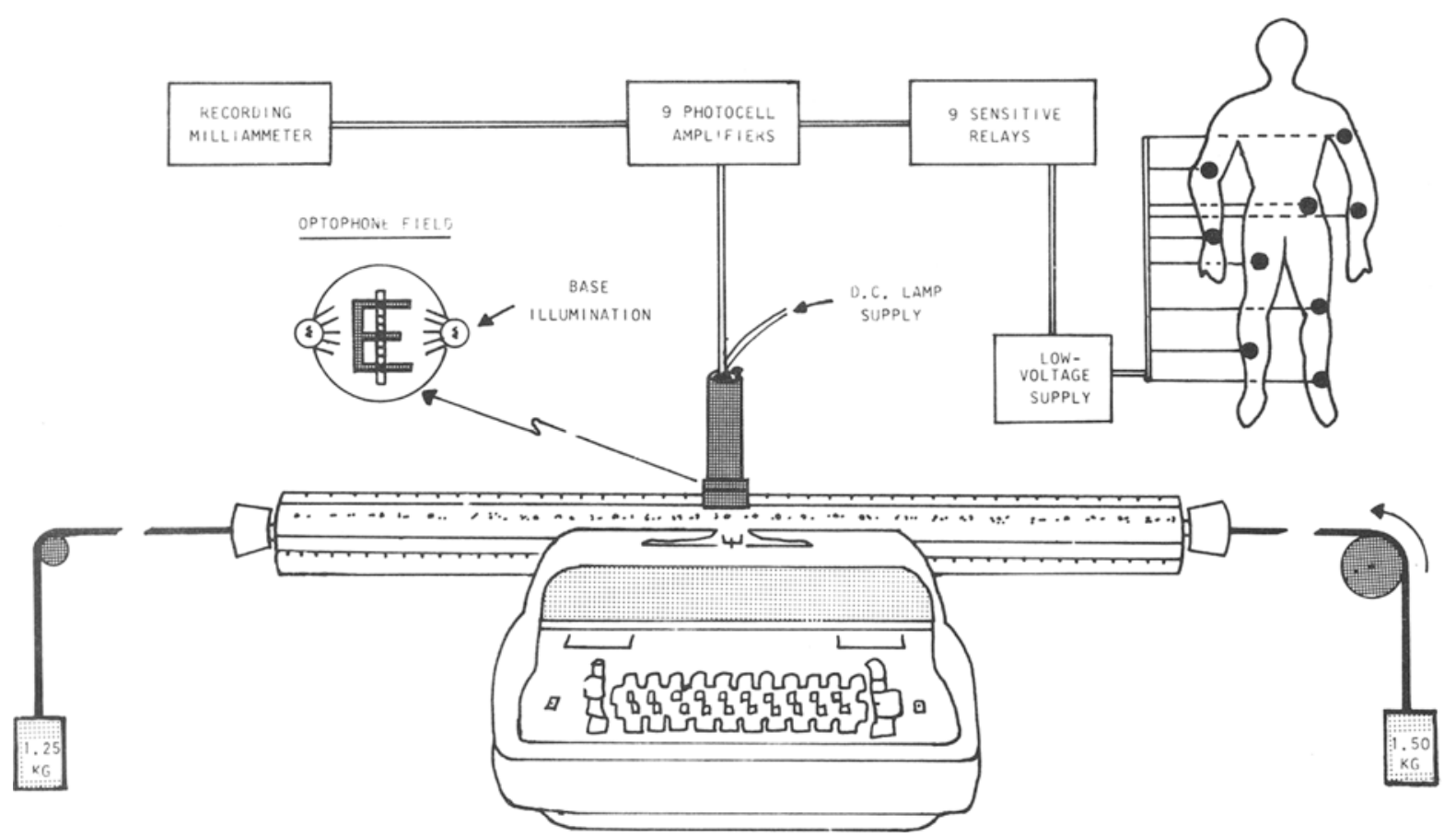

Fig. 2. The optohapt system. The typewriter carriage is driven past the optophone head at a steady rate, optimally to permit 70 characters (including spaces) to be "read" per minute.

learning, come to "mean" the letters and words being surveyed. It is, of course, too early to judge the probable outcome, except that one cannot escape the conclusion that such a device asks a good deal of somesthetic perception.

Meanwhile, there has been devised, in the Princeton Laboratory, the optohapt, also based on photoelectric sensing of printed or typed characters and with a tactual, rather than auditory, output. The optohapt"a Greek remark," to quote W. S. Gilbert, just as "visotactor" is a Latin one-utilizes the probe of the Battelle instrument but treats the nine tiny photocells as variable resistors in nine simple transistor amplifier circuits which operate sensitive relays. These, in turn, control nine vibrators of the Sherrick type (Sherrick, 1965), located as shown in Fig. 1: two on each arm, two on each leg, and one on the abdomen. Loci are carefully chosen to avoid corresponding bodily points and are distributed as widely as possible over the skin surface while avoiding substrates that might, through bone conduction, call the cochlea into play. Figure 2 is an attempt to describe the main features of the optohapt system. ${ }^{2}$

The materials to be "read" are typed directly on low-gloss paper cemented to the platen of a 30-in. IBM electric typewriter of the accounting variety. The typewriter itself does the printing but, more importantly, it also provides the "reader" mechanism. The machine's carriage is carefully weighted at each end to override the typewriter's own drive and to give smooth, steady motion, the speed of which is controlled by a small dc motor operating through a gear-reduction box. The optophone head is thus stationary, while the material to be scanned remains in strict registration as it passes through the viewing area.

A great variety of symbols can be obtained by utilizing the IBM library of type faces. The main characters selected were the serifless capitals of "Manifold" type, but to these were added the full range of punctuation marks, numerals, and many other literary and business symbols. Altogether, the machine provides 86 such characters; additionally, it is equipped with a substitutable type-bar. Many extra symbols thus presented themselves for tryout.

In the first rough search for distinctive materials it became apparent that the temporal features of the signal were likely to be more important than the spatial ones. It also appeared that "unitariness" of the signal would be easily lost either if timing of the total pattern were not right or if the symbol were to be too "busy." Accordingly, much effort went into ascertaining the optimal presentation speed. At least without considerable prior familiarity with the material, it appears that a rate of 70 characters per minute is about "right" in that the majority of usable symbols create, at this speed, lively and somesthetically interesting signals, much of their attention-demandingness coming from the vivid synthetic tactual movement they generate.

It was not necessary to go about the preliminary sorting of symbols in a strictly empirical manner. Some simple engineering considerations enter to permit the elimination of characters yielding highly similar outputs. Thus, if one takes readings, with a recording milliammeter, of the overall current passing through 
the common leg of the nine amplifier circuits, it is possible to see something of the way in which the signal distributes through time. Though the visual properties of the symbols do not necessarily suggest it, the records of distribution of current consumption over time show great similarity of signal pattern among the letters $B, H, M, O, U$, and $X$. All six yield graphic records falling in the "large, bimodal" class. Similar$1 y$, there are many symbols, chiefly punctuation marks, classifiable as "small, unimodal," others (J, T, L) "large, unimodal," still others $(\#, \nabla, \sqrt{ })$ "small, bimodal, " while a few $(@$, , $九$ ) are "small, multimodal" and a few $(W, \Phi)$ are "large, multimodal." Symbols that would inevitably get confused with each other could be discarded on the basis of such information, and it was possible to reduce the candidates for inclusion in a vibrotactile alphabet to 40 without further tryout.

The remaining candidates were then entered into a single pair comparison program of 780 items randomly intermixed. Each pair of symbols, presented in immediate succession, was judged with respect to degree of difference displayed. A simple fourpoint scale was used: 3= "easily distinguished from each other, would never be confused when felt successively"; $0=$ "cannot tell the two apart."

A summary of such judgments, for two practiced observers, is given in Table 1. For each of the 40 symbols, there has been calculated a "coefficient of discriminability" $\left(\mathrm{K}_{\mathrm{D}}\right)$. This is a simple ratio between the total score received by the symbol on the $39 \mathrm{com}-$ parisons and the total possible score (a " 3 " on every pairing).

It is obvious, from a perusal of Table 1 , that it is

Table 1. Relative standing of the 40 "most promising" symbols with respect to discriminability. $K_{D}$ is defined in the text.

\begin{tabular}{|c|c|c|c|c|c|}
\hline SYMBOL & RANK & $\underline{k}_{D}$ & SYMBOL & RANK & $\underline{K}_{0}$ \\
\hline - & 1.5 & .91 & E & 21 & .73 \\
\hline$\equiv$ & 1.5 & .99 & $>$ & 23.5 & .72 \\
\hline . & 2.5 & .90 & B & 23.5 & .72 \\
\hline - & 2.5 & .90 & 0 & 23.5 & .72 \\
\hline 11 & 5 & .87 & 奉 & 23.5 & .72 \\
\hline$=$ & 7 & .85 & 0 & 27.5 & .71 \\
\hline 口 & 7 & .85 & $\neq$ & 27.5 & .71 \\
\hline$\rightarrow$ & 7 & .85 & J & 27.5 & .71 \\
\hline ' & 10 & .82 & $\uparrow$ & 27.5 & .71 \\
\hline : & 10 & .82 & $z$ & 30 & .70 \\
\hline$T$ & 10 & .82 & $\Omega$ & 31.5 & .69 \\
\hline $\mathrm{F}$ & 12 & .81 & $p$ & 31.5 & .69 \\
\hline$\div$ & 13 & .80 & $w$ & 34 & 67 \\
\hline- & 14 & .79 & I & 34 & .67 \\
\hline$L$ & 15.5 & .78 & $<$ & 34 & .67 \\
\hline 口 & 15.5 & .78 & $x$ & 36.5 & .66 \\
\hline I & 17.5 & .76 & $\varepsilon$ & 36.5 & .66 \\
\hline п & 17.5 & .76 & v & 38.5 & .65 \\
\hline$\%$ & 19 & .75 & 2 & 38.5 & .65 \\
\hline 1 & 20 & .74 & A & 40 & .64 \\
\hline
\end{tabular}

not the letters of the alphabet that constitute the promising materials for the optohaptbut rather simpler symbols, punctuation marks especially. By and large, it is the relatively uncomplicated characters, those that yield "small, unimodal" current distribution curves, that get the high ratings. In the first ten ranks there is included not a single letter. Of the 40 symbols to which the list had been narrowed, alphabetic characters ranked 25th, on the average, and the letter "A" was last on the list. Vowels averaged out at 26th. Clearly, it is indicated that "raw" verbal materials are not the most efficacious for this purpose; coding of some kind is needed if the natural proclivities of the tactual sense are to be appealed to by the optohapt.

It would have been possible at this point to take a direct step to the selection of 26 readily available symbols for alphabetic coding. There would only be necessary assignment of the most readily discriminated signals to the most frequently encountered letters in common English prose to have a code of some utility. However, it also has seemed wise to exhaust all avenues of improvement in discriminability before taking the final coding step. To that end, the following have proved feasible:

1. Through the addition of a suitable auxiliary optical system, one which trains on the typewriter platen a medium-power microscope and is equipped with a filar micrometer ocular, it is possible to move a symbol from its normally printed position to a radically different one and thus secure a new signal. The period, which ordinarily triggers the vibrator in the ankle position, can be made to operate in the upper arm position, and this gives two distinctive signals. While they are indiscriminable temporally, their extreme spatial separation gives both great distinctiveness. Comparable things may be done with the hyphen, apostrophe, quotes, etc.

2. Superposition of characters produces new symbols (as, indeed, is known to every child who has made up files of toy soldiers on a typewriter!). An especially effective signal is given by the superposition of $U$ and $T$ (D) giving as it does three closely spaced jabs that involve most of the entire array of vibrators. This source of new characters is more limited than might be supposed; most such inventions are too "busy" and must be rejected along with symbols like@ and Ж as relatively lacking in integrity.

3. A third form of tampering with the typed symbols is to make judicious use of India ink "fill-ins" in preparing the copy. The equality sign operates more reliably as a solid black character than when printed normally as two horizontal parallel lines, and there are numerous other instances.

4. One may go outside the list of available type faces by devising entirely new characters. These may be formed through the expedient of typing in red (to which the photocells are blind) a symbol which provides a set of guidelines for printing, freehand in India ink, a novel 
Table 2. A tentative selection of symbols for alphabetic coding. Letters are arranged in decreasing order of frequency of use in English prose.

\begin{tabular}{|c|c|c|c|c|}
\hline$E$ & . & $\mathrm{L}$ & - & $P$ \\
\hline $\mathrm{T}$ & • & $D$ & "I & $B$ \\
\hline$A$ & " & $U$ & 1 & V \\
\hline 0 & 口 & $C$ & I & $\mathrm{K}$ \\
\hline 1 & 1 & $M$ & Ш & $x$ \\
\hline $\mathrm{N}$ & - & $\mathrm{F}$ & - & $\mathrm{J}$ \\
\hline S & $\diamond$ & $\mathrm{G}$ & 㠷 & $z$ \\
\hline $\mathrm{R}$ & $\Pi$ & $W$ & 1 & $Q$ \\
\hline $\mathrm{H}$ & $\div$ & $Y$ & : & \\
\hline
\end{tabular}

set of marks. Thus, the percent sign (\%) may have its tiny circles filled in, while the slant is disregarded. This gives a vivid signal which first strikes the upper part of the body, then jumps to the legs.

There is one other consideration that enters into the selection of symbols for final coding, viz., the reliability of performance on the part of the photocell head. For nine tiny cells to respond precisely in a system designed for some flexibility with respect to adjustment to type size, there must be no "slippage" in optical registration. Each character must give a positive suprathreshold response in the photocells it is supposed to activate. Certain otherwise desirable symbols have had to be dropped because their reliability in this respect was low. The equivalence sign ( $\equiv$ ) was one of these; the addition sign $(+)$ was another. A few other characters fall in this ca+egory.

Putting together, now, all that has been learned about "good" and "poor"' materials to feed the optohapt, it is possible to devise, at least tentatively, a workable code. Whereas it may prove, in extensive empirical testing, that some minor modifications will ultimately be needed, the alphabetic coding shown in Table 2 seems to possess no major faults. At least, observers are able to recognize and put a name to each of the signals after a little preliminary familiarization. Whether awkward combinations will be found when once the basic alphabet has been learned and rapid sequences of letters and words are exposed remains to be seen. But there are already some indications that other principles, e.g., grouping into larger Gestalten and "following behind" as in other code learning, will come to the rescue to obviate the imperfections.

\section{References}

Bice, R. C. Electromechanical transducer for vibrotactile stimulation. Rev. sci. Instrum., 1961, 32, 856-857.

Foumier d'Albe, E. E. On a type-reading optophone. Proc. Roy. Soc., Lond., 1914, 90A, 373-375.

Freiberger, H. Problems and accomplishments in sensory aids for the blind. Trans. N. Y. Acad. Sci., 1965, 27, 414-421.

Geldard, F. A. Some neglected possibilities of communication. Science, 1960, 131, 1583-1588.

Geldard, F. A., \& Sherrick, C. E. Multiple cutaneous stimulation: the discrimination of vibratory patterns. J. Acoust. Soc. Amer., $1965,37,797-801$.

Pratt, C. C. The spatial character of high and low tones. J. exp. Psychol., 1930, 13, 278-285.

Rousseau, J. J. Emile, ou de L'Education. Tome VII, Oeuvres de Jean Jaques Rousseau. Amsterdam: Néaulme, 1762.

Sherrick, C.E. Simple electromechanical vibration transducer. Rev. sci. Instrum., 1965, 36, 1893-1894.

Smith, G. C., \& Mauch, H. A. The development of a reading machine for the blind. Washington: VA Dept. Med. Surg., June, 1964.

\section{Notes}

1. The work reported was supported, in part, by grant GB-1020 from the National Science Foundation and, in part, by grant NB04755 from the National Institutes of Health, U. S. Department of Health, Education, and Welfare.

2. Thanks are due the Research and Development Division, Prosthetic and Sensory Aids Service, Veterans' Administration, for the loan of the Battelle Optophone unit used in this system.

(Accepted for publication September 29, 1966.) 